\title{
Espèces réactives de l'oxygène et stress oxydant
}

> Le stress oxydant se définit par un déséquilibre entre la production d'espèces radicalaires (ou réactives) de l'oxygène ( $\varepsilon R 0)$ et les capacités cellulaires antioxydantes. Les ERO ont longtemps été considérées comme des sous-produits toxiques du métabolisme normal de l'oxygène et impliquées dans de nombreuses pathologies. Cependant, depuis plusieurs années, la production contrôlée de radicaux apparaît comme un mécanisme essentiel de la signalisation cellulaire qui participe au maintien de l'homéostasie de la cellule. Les effets des radicaux libres en biologie sont maintenant bien documentés. Non seulement les organismes vivants se sont adaptés et coexistent en présence de radicaux $\varepsilon R O$ mais ils ont également développé des mécanismes pour les utiliser à leur avantage. Néanmoins, les sources de radicaux et leurs mécanismes d'action sont souvent mal définis. Cette revue fait ainsi le point sur les principales propriétés des $\varepsilon R 0$ et leurs effets paradoxaux. <

Après la découverte des radicaux libres dans les systèmes biologiques il y a un peu plus de 55 ans [1], Harman et al. [2] évoquent pour la première fois en 1956 l'hypothèse selon laquelle l'accumulation des dommages moléculaires et cellulaires causés par les radicaux libres centrés sur l'oxygène serait responsable des phénomènes du vieillissement. Ces radicaux sont par définition des espèces chimiques possédant un électron célibataire sur leur couche périphérique, ce qui leur confère un fort degré de réactivité. Ils ont été longtemps considérés comme nuisibles, responsables de potentiels dommages à l'ADN, aux protéines et aux lipides. Cependant, la description de la production d'anion superoxyde $\left(\mathrm{O}_{2}{ }^{-*}\right)$ par les cellules phagocytaires dans la lutte contre les pathogènes, celle du rôle biologique du monoxyde d'azote (NO) ainsi que la découverte des enzymes productrices qui leur sont associées soulignent également le rôle physiologique du stress oxydant.
Camille Migdal, Mireille Serres

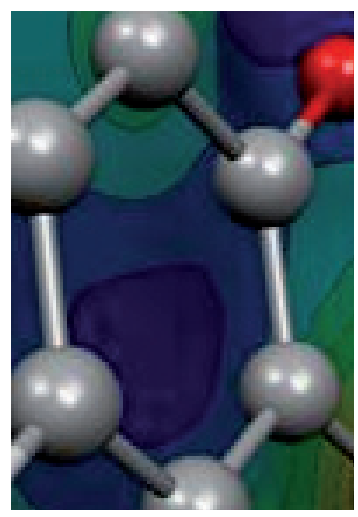

Université Lyon 1, EA 41-69,

Laboratoire de recherche dermatologique, pavillon $\mathrm{R}$, Hôpital Édouard Herriot, 69437 Lyon Cedex 03,

France.

camille.migdal@u-psud.fr

Depuis plusieurs années maintenant, la production contrôlée d'ERO apparaît comme un mécanisme incontournable de la régulation et de la signalisation cellulaires qui participe au maintien de l'homéostasie radicalaire de la cellule. Une perturbation de cette homéostasie est considérée à l'heure actuelle comme impliquée dans le développement de nombreuses pathologies, et la compréhension des mécanismes d'action des $\varepsilon R O$ devient un enjeu clinique.

\section{Origine des espèces réactives de l'oxygène}

\section{Oxygène et chaîne respiratoire mitochondriale}

À l'exception de certains organismes anaérobies et aérotolérants', l'oxygène (ou dioxygène, $\mathrm{O}_{2}$ ) est indispensable à la production d'énergie par de nombreuses formes de vie (animaux, plantes, bactéries). Cette production d'énergie (sous forme d'ATP) appelée phosphorylation oxydative se fait notamment par l'intermédiaire de chaînes de transport d'électrons présentes dans la membrane interne des mitochondries [31]. Ainsi, depuis que l'atmosphère terrestre a commencé à s'enrichir en oxygène il y a environ deux milliards d'années, les organismes vivants aérobies se sont adaptés à ces conditions en apprenant à consommer et à utiliser l'oxygène mais également à éliminer les métabolites réduits produits. En effet, lors du métabolisme normal, la réduction tétravalente de l'oxygène en eau (Figure l) se fait en plusieurs étapes successives qui donnent naissance à des intermédiaires potentiellement réduits, appelés radicaux primaires ou espèces réactives de l'oxygène ( $\varepsilon R 0)$, car ces entités radicalaires et moléculaires sont beaucoup plus réactives que l'oxygène qui leur a donné naissance. Ainsi, environ $2 \%$ de l'oxygène consommé au niveau

${ }^{1}$ Organismes anaérobies par nature mais qui peuvent survivre en présence d'oxygène qu'ils n'utilisent pas. 


\begin{tabular}{|c|c|c|}
\hline $\begin{array}{l}\text { Oxygène } \\
\qquad \mathrm{O}_{2}\end{array}$ & \multicolumn{2}{|c|}{$\begin{array}{l}\text { Superoxyde Réaction de Fenton Catalase } \\
\text { dismutases }\end{array}$} \\
\hline \multicolumn{2}{|c|}{$\begin{array}{l}\text { Réduction tétravalente } \\
\text { de l'oxygène }\end{array}$} & $\mathrm{O}_{2}+4 \mathrm{e}^{-}+4 \mathrm{H}^{+} \rightarrow 2 \mathrm{H}_{2} \mathrm{O}$ \\
\hline $\mathrm{O}_{2} \cdot-$ & Anion superoxyde & $1 \mathrm{O}_{2}+1 \mathrm{e}^{-} \rightarrow \mathrm{O}_{2} \cdot-$ \\
\hline $\mathrm{H}_{2} \mathrm{O}_{2}$ & $\begin{array}{l}\text { Peroxyde } \\
\text { d'hydrogène }\end{array}$ & $2 \mathrm{O}_{2} \cdot-\mathrm{O}_{2} \cdot-\left(+2 \mathrm{H}^{+}\right) \rightarrow \mathrm{H}_{2} \mathrm{O}_{2}+\mathrm{O}_{2}$ \\
\hline$\cdot \mathrm{OH}$ & Radical hydroxyle & $\begin{array}{l}3 \mathrm{H}_{2} \mathrm{O}_{2}+\mathrm{Fe}^{2+} \rightarrow \cdot \mathrm{OH}+\mathrm{Fe}^{3+}+\mathrm{OH}^{-} \\
4 \mathrm{H}_{2} \mathrm{O}_{2}+\mathrm{H}_{2} \mathrm{O}_{2} \rightarrow 2 \mathrm{H}_{2} \mathrm{O}+\mathrm{O}_{2} \\
5 \mathrm{H}_{2} \mathrm{O}_{2}+2 \mathrm{GSH} \rightarrow 2 \mathrm{H}_{2} \mathrm{O}+\mathrm{GSSG}\end{array}$ \\
\hline $\mathrm{RO}_{2}^{*}$ & Radical peroxyle & $6 \mathrm{R}^{\cdot}+\mathrm{O}_{2} \rightarrow \mathrm{RO}_{2}^{\cdot}$ \\
\hline $\mathrm{RO}_{2} \mathrm{H}$ & Hydroperoxyde & $7 \mathrm{RO}_{2}^{-}+\mathrm{RH} \rightarrow \mathrm{RO}_{2} \mathrm{H}+\mathrm{R}^{\cdot}$ \\
\hline RO & Radical alkoxyle & $8 \mathrm{RO}_{2} \mathrm{H}+\mathrm{Fe}^{2+} \rightarrow \mathrm{RO}^{-}+\mathrm{Fe}^{3+}+\mathrm{OH}^{-}$ \\
\hline
\end{tabular}

Figure 1. Origine des espèces réactives de l'oxygène. Les quatre étapes de la réduction de l'oxygène et la formation des intermédiaires partiellement réduits sont détaillées. type « réaction de Fenton », à la plus délétère des espèces radicalaires du stress oxydant, le radical hydroxyle ${ }^{\circ} \mathrm{OH}$ (Figure 1-3). La quantité de peroxyde d'hydrogène est régulée par l'enzyme héminique catalase (CAT) qui accélère sa dismutation (Figure 1-4), et par la glutathion peroxydase (GPx) qui catalyse sa réduction par le glutathion (Figure 1-5). Le glutathion (GSH) est un tripeptide (acide glutamique-cystéineglycine) qui représente le groupement thiol majoritaire au niveau intracellulaire $\left(10^{-4}\right.$ à $10^{-3}$ mol. $\mathrm{L}^{-1}$ sous forme réduite). II possède un fort pouvoir réducteur et peut également chélater les ions $\mathrm{Cu}^{2+}$ et ainsi limiter leur participation à la génération d'ERO par les réactions de type Fenton (Figure 1-3). Toutefois, il existe également d'autres $\varepsilon R O$, dit secondaires, comme les radicaux peroxyles $\mathrm{RO}_{2} \cdot$ (Figure 1-6), les hydroperoxydes $\mathrm{RO}_{2} \mathrm{H}$ (Figure 1-7) et les radicaux alkoxyles RO* (Figure 1-8).

\section{NAD $(P) H$ oxydases et autres sources cellulaires d'ERO}

$\varepsilon$ n parallèle de la production d'ERO par le complexe enzymatique mitochondrial de la chaîne respiratoire, la plupart des cellules sont capables de produire des radicaux superoxydes $\mathrm{O}_{2}{ }^{-}$ via une activité $\mathrm{NAD}(\mathrm{P}) \mathrm{H}$ oxydase membranaire (NOX). La NOX est une enzyme qui catalyse la mitochondrial sont transformés en radicaux superoxydes $\mathrm{O}_{2}{ }^{--}$lors de la première réduction électronique de l'oxygène (Figure 1-1). La dégradation de ces radicaux est contrôlée par des systèmes de défense, les antioxydants, qui s'adaptent au taux de radicaux présents. Ainsi, la production des radicaux superoxydes est régulée par des métalloenzymes, les superoxydes dismutases (SOD), qui catalysent leur dismutation en peroxyde d'hydrogène, $\mathrm{H}_{2} \mathrm{O}_{2}$ (Figure 1-2). Bien que le peroxyde d'hydrogène ne soit pas en soi un radical mais une molécule, il est luimême toxique et capable de donner naissance, via des réactions de réduction monoélectronique de $\mathrm{I}^{\prime} \mathrm{O}_{2}$ en utilisant le NADPH ou le NADH comme donneur d'électrons :

$$
\mathrm{NAD}(\mathrm{P}) \mathrm{H}+2 \mathrm{O}_{2} \Rightarrow \mathrm{NAD}(\mathrm{P})^{+}+\mathrm{H}^{+}+2 \mathrm{O}_{2} \cdot
$$

La NOX a été initialement étudiée dans les cellules phagocytaires où elle joue un rôle primordial dans la défense contre les pathogènes, mais elle existe également dans toutes les autres cellules non phagocytaires où elle participe à la signalisation cellulaire. La NOX est localisée dans la membrane cytoplasmique

Tableau I. Les NADPH oxydases. Toutes les enzymes de la famille des NOX possèdent un domaine gp91 phox/NOX2, ou similaire, composé d'un domaine carboxy-terminal cytoplasmique capable de fixer le $N A D(P) H$ et d'un domaine amino-terminal comportant six domaines transmembranaires ayant deux groupements héminiques (flèche rouge). Les électrons sont transférés du NAD $(P) H$, via le $F A D$ et les hèmes intramembranaires, à l'oxygène moléculaire, donnant naissance au radical superoxyde $0_{2}{ }^{*}$. Les $N O X 2$ et $1,3,4$ forment un hétérodimère avec p2 $2^{\text {phox }}$. Des homologues des protéines cytosoliques régulatrices de NOX2, NOXAl et NOXOl sont impliqués dans l'activité des NOX1, 3 et 4. NOX5 et les Duoxl et 2 possèdent des domaines dits « mains $\varepsilon-F »$, qui rendent leur activité oxydase calcium dépendante. Enfin, les Duox peuvent produire $\mathrm{H}_{2} \mathrm{O}_{2}($ d'après $[5,6])$.

Erk, extracellular signal regulated kinase; FGF, fibroblast growth factor; IGF, insulin-like growth factor 1 ; IL, interleukin; IP3, inositol trisphosphate ; JNK, c-Jun N-terminal kinase ; LPS, lipopolysaccharide; MAPK, mitogen-activated protein kinase ; MAPKAPK2 ; MAPK activated kinase-2 ; MKP, MAPK phosphatase; MMP, matrix metalloproteinase; PAK1, p21-activated protein kinase; PDGF, platelet-derived growth factor; PDK1, phosphoinositide-dependent kinase-1 ; pRb, retinoblastoma protein ; SSH1L, slingshot $1 L$ phosphatase ; STAT, signal transducers and activators of transcription; TGF, transforming growth factor; TNF, tumor necrosis factor ; TRPV1, transient receptor potential vanilloid receptor 1 ; VEGF, vascular endothelial growth factor. n.d., non déterminé. 


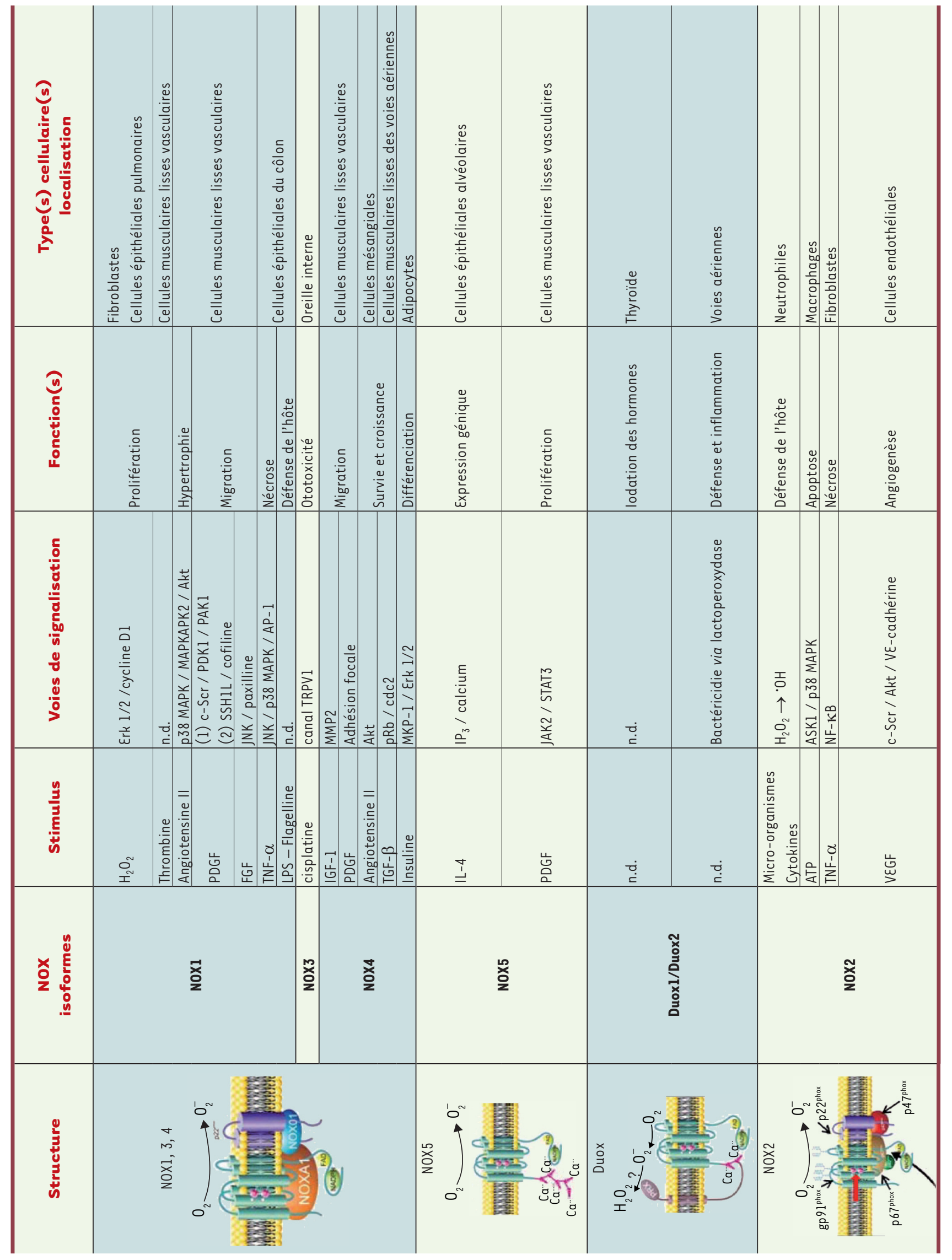




\begin{tabular}{|c|c|}
\hline $\begin{array}{c}\text { Radicaux } \\
\text { Caractéristiques }\end{array}$ & Mode d'action \\
\hline $\begin{array}{l}\text { Radical superoxyde } \mathbf{0}_{2}{ }^{\cdot-} \\
\text { - Constante de vitesse faible } \\
\text { - Très peu réactif } \\
\text { - Toxicité indirecte }\end{array}$ & 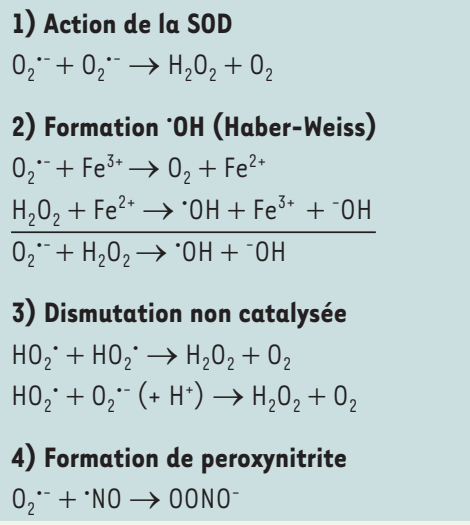 \\
\hline $\begin{array}{l}\quad \text { Radical hydroxyle } \mathbf{O H} \\
\text { - Constante de vitesse élevée } \\
\text { - Réaction limitée par la diffusion } \\
\text { - Pas de cible privilégiée } \\
\text { - Faible durée de vie }\end{array}$ & $\begin{array}{l}\text { 1) Arrachement d'un électron } \\
\cdot \mathrm{OH}+\mathrm{Fe}^{2+} \rightarrow-\mathrm{OH}+\mathrm{Fe}^{3+} \\
\text { 2) Arrachement d'un atome d'hydrogène } \\
\cdot \mathrm{OH}+\mathrm{RH} \rightarrow \mathrm{R}^{\cdot}+\mathrm{H}_{2} \mathrm{O} \\
\text { 3) Addition sur des doubles liaisons } \\
\cdot \mathrm{OH}+>\mathrm{C}=\mathrm{C}<\rightarrow>\cdot \mathrm{C}-\mathrm{C}(\mathrm{OH})-\end{array}$ \\
\hline
\end{tabular}

Tableau II. Mode d'action des radicaux libres primaires.

et dans certains granules spécifiques des neutrophiles. Selon le type cellulaire, la NOX peut libérer $\mathrm{O}_{2}{ }^{*-}$ de la cellule, de manière préférentielle vers l'extérieur (cellules phagocytaires) ou vers l'intérieur (cellules non phagocytaires). Parmi les isoformes existant dans la famille NOX (Tableau I), la NOX2 des cellules phagocytaires est la plus étudiée. Les sous-unités principales constitutives de la NOX2 sont présentes dans deux compartiments cellulaires distincts : la membrane (où se trouve le site catalytique, le cytochrome $b_{558}$ composé de gp9 $1^{\text {phox }}$ et $\mathrm{p} 22^{\text {phox }}$ ) et le cytosol (où sont localisées les protéines régulatrices $p 47^{\text {phox }}, p 40^{\text {phox }}$ et $p 67^{\text {phox }}$ ). Après stimulation, les protéines cytosoliques migrent vers la membrane et s'associent au cytochrome $b_{558}$ pour former une NOX2 active par l'intermédiaire de protéines $G$ [3]. Les autres isoformes de la NOX (NOXI, 3, 4, 5 et Duoxl et 2 ; voir Tableau l) partagent toutes une homologie de séquence avec gp9 $1^{\text {phox }}[3,4]$. Les $N O X 1,3$ et 4 forment un hétérodimère avec $p 22^{\text {phox }}$, et des homologues des protéines cytosoliques régulatrices de NOX2 ( $44^{\text {phox }}$ et $p 67^{\text {phox }}$ ), appelés NOXAl et NOXO1, impliqués dans leur activité. NOX5 et les Duoxl et 2 possèdent des structures supplémentaires, des domaines dits « mains $\varepsilon-F »^{2}$, qui rendent leur activité oxydase dépendante du calcium. Enfin, les Duox possèdent une très longue région extracellulaire ayant une forte homologie de séquence avec les peroxydases et peuvent produire $\mathrm{H}_{2} \mathrm{O}_{2}$ [4].

${ }^{2}$ Ces sites mis en évidence pour la première fois dans la parvalbumine, puis dans la calmoduline ont une structure trés particulière appelée $\varepsilon F-h a n d$, sous forme d'un motif supersecondaire hélice-boucle-hélice. La boucle va loger un atome de calcium.
En plus des NOX, d'autres sources, cytosoliques ou présentes au sein de différents organites, peuvent produire des $\varepsilon R O$. Par exemple, la xanthine oxydase, qui catalyse l'oxydation de l'hypoxanthine et de la xanthine au cours du métabolisme des purines, entraîne la formation $\mathrm{d}^{\prime} \mathrm{O}_{2}{ }^{*}$. Les enzymes du réticulum endoplasmique, dont la famille des cytochromes $\mathrm{P}_{450}$, assurent l'oxydation des acides gras insaturés (et de certains xénobiotiques) et réduisent l'oxygène moléculaire pour former $\mathrm{O}_{2}{ }^{*-}$ et/ou $\mathrm{H}_{2} \mathrm{O}_{2}$. Les enzymes de la voie de l'acide arachidonique, les peroxysomes, les lysosomes (qui contiennent la myéloperoxydase responsable de la formation d'acide hypochloreux) et le noyau (qui possède des cytochromes oxydases et une chaîne de transport d'électrons) sont également capables de produire $\mathrm{O}_{2}^{\cdot-}[7]$.

\section{Caractéristiques des \&RO et modes d'action}

Le radical superoxyde $\mathrm{O}_{2}{ }^{--}$est l'ERO qui possède la plus faible réactivité vis-à-vis des substrats bio-organiques en raison, notamment, d'une constante de vitesse faible. On lui connaît quelques cibles privilégiées comme le cytochrome c $\left(\mathrm{Fe}^{3+}\right)\left(\mathrm{k}=2,6 \times 10^{5} \mathrm{~mol}^{-1} \cdot \mathrm{L} \cdot \mathrm{s}^{-1}\right)$, la vitamine $\mathrm{C}$ $\left(\mathrm{k}=2,7 \times 10^{8} \mathrm{~mol}^{-1} \cdot \mathrm{L} \cdot \mathrm{s}^{-1}\right)$, et bien entendu la SOD $\left(\mathrm{k}=2 \times 10^{9} \mathrm{~mol}^{-1} \cdot \mathrm{L} \cdot \mathrm{s}^{-1}\right)$. Cependant, $\mathrm{I}^{\prime} \mathrm{O}_{2}{ }^{--}$est capable de produire d'autres radicaux beaucoup plus réactifs. II s'agit de toxicité indirecte (Tableau II) :

- via la réaction d'Haber-Weiss, catalysée par le $\mathrm{Fe}^{3+}$, qui traduit la formation de radicaux hydroxyles ${ }^{\circ} \mathrm{OH}$ lors de la réduction de $\mathrm{H}_{2} \mathrm{O}_{2}$;

- via l'intervention de la forme protonée de l' $\mathrm{O}_{2}{ }^{*}$, le radical perhydroxyle $\left(\mathrm{HO}_{2}{ }^{\circ}\right)$, qui semblerait être la forme dite active. En effet, $\mathrm{HO}_{2}{ }^{\cdot}$ possède des constantes de vitesse plus importantes, vis-à-vis notamment des acides gras polyinsaturés $\left(10^{3} \mathrm{~mol}^{-1} \cdot \mathrm{L} \cdot \mathrm{s}^{-1}\right)$ avec lesquels $\mathrm{O}_{2}{ }^{*-}$ ne réagit pas. $\mathrm{HO}_{2} \cdot$ participe notamment à la dismutation non catalysée de $\mathrm{O}_{2}{ }^{\circ-}$, c'est-à-dire en l'absence de SOD ;

- via la réaction $\mathrm{d}^{\prime} \mathrm{O}_{2}{ }^{--}$avec le monoxyde d'azote radicalaire 'NO qui donne naissance au peroxynitrite $00 \mathrm{NO}^{-}$, bien connu pour ses effets délétères sur l'ADN, les protéines et les lipides.

Le radical hydroxyle ${ }^{\circ} \mathrm{OH}$ est l'oxydant le plus puissant des $\varepsilon R O$ avec une constante de vitesse élevée. À titre d'exemple, $\mathrm{k}(\cdot \mathrm{OH}+\mathrm{ADN})=4 \times 10^{8} \mathrm{~mol}^{-1} \cdot \mathrm{L} \cdot \mathrm{s}^{-1}$; $\mathrm{k}(\cdot \mathrm{OH}+$ cystéine $)=4 \times 10^{10} \mathrm{~mol}^{-1} \cdot \mathrm{L} \cdot \mathrm{s}^{-1} ; \mathrm{k}(\cdot \mathrm{OH}+\mathrm{glu}-$ cose $)=7,4 \times 10^{8} \mathrm{~mol}^{-1} \cdot \mathrm{L} \cdot \mathrm{s}^{-1}$. Ces réactions sont cependant limitées par la diffusion, et $\cdot \mathrm{OH}$ est donc une espèce qui réagit avec des substrats sur son lieu de production, qui ne possède pas de cibles privilégiées et 


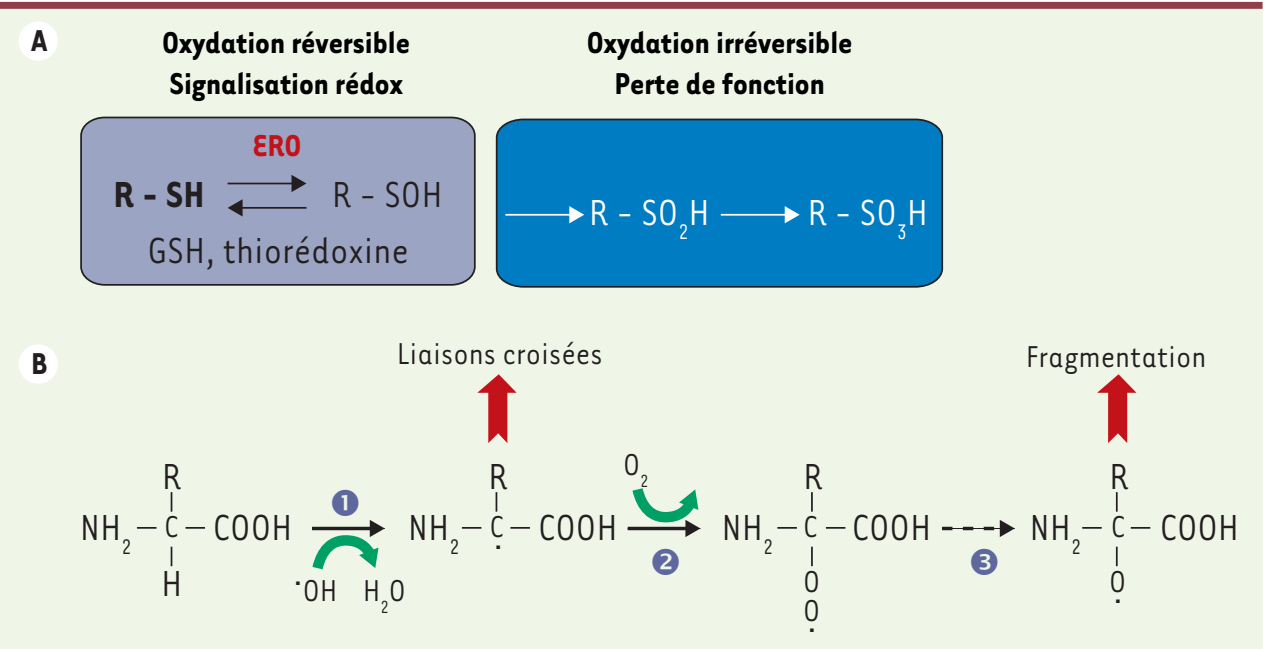

Figure 2. Attaque radicalaire des protéines. A. Oxydation des fonctions thiols de la cystéine. Les résidus cystéines peuvent être oxydés en acide sulfénique ( $\mathrm{R}-\mathrm{SOH})$, puis en acide sulfinique $\left(\mathrm{R}-\mathrm{SO}_{2} \mathrm{H}\right)$ et sulfonique $\left(\mathrm{R}-\mathrm{SO}_{3} \mathrm{H}\right)$. Alors que les acides sulfiniques et sulfoniques sont stables et que leur oxydation est irréversible, la formation d'acide sulfénique est réversible sous l'action du glutathion et/ou de la thiorédoxine. B. Oxydation de la chaîne polypeptidique. 1. L'oxydation est initiée par le radical hydroxyle qui enlève un atome d'hydrogène sur le carbone alpha d'une liaison peptidique en donnant naissance à un radical centré sur le carbone. En l'absence d'oxygène, deux radicaux centrés sur le carbone peuvent réagir ensemble pour former des liaisons croisées intra ou interchaînes. 2. En présence d'oxygène, une réaction d'addition a lieu pour former un radical peroxyle. 3. Après une série de réactions complètes, un radical alkoxyle est formé, étape nécessaire à la fragmentation de la chaîne polypeptidique (d'après [11]).

a une très faible durée de vie. ${ }^{\circ} \mathrm{OH}$ peut oxyder un substrat selon trois modes d'action : arrachement d'un électron, arrachement d'un atome d'hydrogène sur un substrat organique ou addition sur une double liaison (Tableau II). Les deux derniers modes d'action conduisent à la formation de radicaux centrés sur le carbone.

\section{Stress oxydant}

\section{Définition}

Les $\varepsilon R 0$ sont présentes dans la cellule à des doses raisonnables: leur concentration est régulée par l'équilibre entre leur taux de production et leur taux d'élimination par les systèmes antioxydants [8]. Ainsi, à l'état quiescent, on dit que la balance antioxydants/pro-oxydants (balance rédox) est en équilibre. Cependant cette homéostasie rédox peut être rompue, soit par une production excessive d'ERO (comme dans le vieillissement ou l'athérosclérose), soit par une diminution des capacités antioxydantes (comme chez les personnes souffrant d'obésité et les fumeurs). On parle alors de stress oxydant. Un tel déséquilibre peut être provoqué de façon régulée par l'activation de systèmes de production d'ERO. La réponse antioxydante est alors efficace pour compenser cette production et le déséquilibre est transitoire. En revanche, dans certaines situations pathologiques (cancer), la production d'ERO est plus importante et prolongée, et la réponse antioxydante insuffisante. Le déséquilibre est durable. Cette rupture de l'homéostasie rédox peut avoir plusieurs origines: stress d'origine exogène (agents environnementaux pro-oxydants), intoxication aux métaux lourds, irradiations, carence en antioxydants apportés par l'alimentation ou anomalies génétiques.

\section{ERO : le paradoxe}

Le paradoxe des ERO est qu'elles constituent des produits potentiellement toxiques du métabolisme et sont en même temps des molécules essentielles à la signalisation et à la régulation cellulaires. Cette double potentialité peut être expliquée, au moins en partie, par le fait que les réponses cellulaires varient de manière très sensible et très différente en fonction de la nature chimique de l'ERO, de son niveau de production (effet dose-dépendant) ou encore du site producteur.

Une production modérée et contrôlée d'ERO peut entraîner une oxydation réversible des molécules environnantes: les $\varepsilon R 0$ agissent alors en véritables seconds messagers. À l'inverse, une surproduction d'ERO ou un déficit des systèmes de défense entraîne l'apparition d'un stress qui provoque l'oxydation, de manière non spécifique et irréversible de molécules biologiques, conduisant à une perte de fonction [9]. De nombreuses molécules sont susceptibles d'être attaquées ou modifiées par les ERO. Les acides aminés et les protéines sont les premières cibles. On a estimé que $50 \%$ à $70 \%$ des $\varepsilon$ RO produites par une cellule peuvent être piégées par les protéines. Les modifications oxydatives créées peuvent être de plusieurs types:

- oxydation des chaînes latérales des acides aminés. Tous les acides aminés sont des cibles potentielles pour les ERO [10]; néanmoins les cibles majeures sont les acides aminés soufrés (cystéine, méthionine), basiques (arginine, histidine, lysine) et aromatiques (phénylalanine, tyrosine, tryptophane). Citons par exemple la cystéine (Figure 2A) dont les 
oxydations réversibles jouent un rôle important dans la régulation de la fonction de nombreuses protéines [12];

- oxydation de la chaîne polypeptidique potentiellement suivie d'une fragmentation et/ou de la formation de liaisons croisées intra ou inter chaînes (Figure 2B);

- formation de protéines carbonylées (issues de la fragmentation de chaînes polypeptidiques, de l'oxydation de certains acides aminés ou de l'interaction de leur chaîne latérale avec des produits de la peroxydation lipidique). Ainsi, l'oxydation de protéines spécifiques n'est pas uniquement le témoin de la présence d'un stress oxydant délétère mais intervient également dans la régulation de processus métaboliques [13].

Citons également une autre cible majeure de l'attaque radicalaire : les acides gras polyinsaturés, comme l'acide linoléique et l'acide arachidonique, présents notamment dans les membranes plasmiques. Leur oxydation par les $\varepsilon R 0$, appelée peroxydation lipidique, conduit à la formation de très nombreux produits primaires (les hydroperoxydes) et secondaires (les aldéhydes) dont les activités biologiques sont multiples [14].

\section{Les $\varepsilon R 0$ : bénéfiques ou délétères ?}

\section{Les effets bénéfiques : l'exemple de la défense antimicrobienne}

Un grand nombre de fonctions physiologiques sont sous le contrôle des $\varepsilon R O$ et de leurs effets activateurs/régulateurs dans les voies de signalisation. De nombreux ligands peuvent être à l'origine d'une production d'ERO : des facteurs de croissance comme le PDGF (platelet-derived growth factor) ou l'EGF (epidermal growth factor), des cytokines telles que I'INFy (interféron $\gamma$ ) ou le TNF $\alpha$ (tumor necrosis factor $\alpha$ ) et des peptides comme l'angiotensine II. Ces ligands se fixent à leurs récepteurs spécifiques (récepteurs des cytokines, récepteurs à activité tyrosine kinase, sérine/ thréonine kinase, récepteurs couplés aux protéines $G$ ou à des canaux ioniques) et induisent des ERO qui participent alors à la transduction et à l'amplification du signal. On parle de signalisation rédox : les $\varepsilon R O$ induites peuvent activer des récepteurs par phosphorylation, inhiber des phosphatases amplifiant ainsi les cascades de signalisation, activer directement des kinases (MAPK ou mitogen activated protein kinase, $\mathrm{PKC}$ ou protéine kinase $\mathrm{C}$ ) et des facteurs de transcription ( $A P-1, N F-\kappa B$ ) ou déclencher une augmentation du calcium cytosolique (pour revue, voir [15]). Les ERO amplifient et/ou induisent les signaux intracellulaires par modification de l'équilibre rédox intracellulaire (équilibre GSH/ GSSG et thiorédoxine capable de moduler le signal rédox intracellulaire) et par modification oxydative des protéines. Par exemple, dans les cellules musculaires lisses, le PDGF induit une production transitoire d' $\mathrm{H}_{2} \mathrm{O}_{2}$ et $\mathrm{d}^{\prime} \mathrm{O}_{2}{ }^{-*}$ via un mécanisme dépendant de NOX5 [16-18] qui entraîne l'activation des voies de signalisation EROdépendantes comme les MAPK, NF- $K B$ et JAK/STAT (Janus kinase/ signal transducer and activator of transcription), responsables in fine de l'activité mitogène du PDGF (platelet-derived growth fac- tor) (Tableau I). Dans le cas où le ligand est l'EGF, la production d' $\mathrm{H}_{2} \mathrm{O}_{2}$ induite requiert l'activité kinase de son récepteur à activité tyrosine kinase (mais pas son autophosphorylation) et entraîne l'inhibition de l'activité tyrosine phosphatase $[15,19]$. La production d'ERO induite par la fixation de ces facteurs de croissance et de certaines cytokines à leurs récepteurs se fait notamment par l'intermédiaire de la protéine G Racl [15, 20].

Ainsi, parmi les fonctions physiologiques à composante radicalaire, on peut citer : la régulation du tonus vasculaire, la relaxation du muscle lisse, l'adhésion plaquettaire, la régulation des fonctions contrôlées par la concentration en oxygène et l'apoptose [15]. L'exemple le plus pertinent du rôle physiologique des $\varepsilon R O$ est celui de la défense antimicrobienne (bactéricidie dépendante de l'oxygène) qui fait intervenir trois systèmes enzymatiques pour la formation d'ERO et d'ERA (espèces réactives de l'azote) [21]. Juste après la phagocytose du pathogène, les phagocytes augmentent considérablement leur consommation d'oxygène et passent d'un état quiescent à un état activé. L'oxygène est réduit par la NOX2 phagocytaire en radical superoxyde $0_{2}{ }^{--}$, qui est lui même bactéricide, et par formation d'autres espèces comme le peroxyde d'hydrogène. $\mathrm{H}_{2} \mathrm{O}_{2}$, à son tour, en présence de métaux de transition, peut donner naissance au puissant radical hydroxyle $\cdot \mathrm{OH}$. En parallèle, les myéloperoxydases (MPO), en milieu acide, catalysent l'oxydation des ions halogénures par $\mathrm{H}_{2} \mathrm{O}_{2}$ pour former l'acide hypochloreux $(\mathrm{HOCl})$. $\varepsilon \mathrm{t}$ enfin, les iNOS (NO synthases inductibles) forment des ERA et notamment du monoxyde d'azote radicalaire 'NO (très important pour la lyse des bactéries et des parasites intracellulaires) qui, en réagissant avec $\mathrm{O}_{2}{ }^{--}$produit par NOX2, donne naissance au peroxinitrite $00 \mathrm{NO}^{-}$. Toutes ces $\varepsilon R 0 / \varepsilon R A$ qui interagissent les unes avec les autres afin de détruire le pathogène traduisent l'explosion oxydative.

\section{Les effets délétères :}

\section{l'exemple de l'athérosclérose et des anthracyclines}

Par ailleurs, bien que les ERO participent à de nombreuses fonctions physiologiques, elles contribuent également à la pathogenèse d'un grand nombre de maladies qui possèdent un caractère radicalaire (diabète sucré, maladies cardiaques et neurodégénératives, pathologies articulaires, cancérogenèse, vieillissement). Citons par exemple l'implication des ERO dans l'athérosclérose. L'athérosclérose est une pathologie inflammatoire chronique et multifactorielle [22], définie par un remaniement de l'intima 
des grosses et moyennes artères conduisant à la formation de plaques athéromateuses [23]. La théorie oxydative de l'athérosclérose décrit l'importance des $\varepsilon R O$ dans la formation de ces plaques. En effet, les LDL (low density lipoprotein) circulantes diffusent dans la paroi vasculaire où elles vont subir des modifications oxydatives. Ces LDL oxydées perturbent alors les fonctions des cellules endothéliales, des monocytes/macrophages infiltrés et résidents et des cellules musculaires lisses [24]. Il existe d'ailleurs une réelle relation entre l'incidence des accidents coronariens et le taux plasmatique de cholestérol-LDL. De plus, de nombreux facteurs de risque de l'athérosclérose sont des pro-oxydants, augmentant la production d'ERO par les cellules et créant ainsi un environnement oxydant dans la paroi artérielle.

Citons enfin le rôle des $\varepsilon R O$ dans la toxicité des anthracyclines. La doxorubicine (DOX) est l'une des drogues anticancéreuses les plus utilisées et les plus performantes. Elle peut cependant entraîner, à fortes doses cumulatives, des troubles cardiaques [25]. Bien que les mécanismes moléculaires responsables de la mort des cellules cardiaques restent encore controversés (de très nombreuses voies de signalisation ont été décrites), l'induction d'un stress oxydant par la DOX est la principale et la plus répandue des hypothèses (revue dans [26]). Cette production d'દRO s'explique notamment par la structure de la DOX qui contient une quinone capable de subir un cycle rédox, entraînant la formation $d^{\prime} \mathrm{O}_{2}{ }^{\cdot-}$, et par la présence de fer qui catalyse la formation d' $\mathrm{OH}$ [27]. Ces $\varepsilon R O$ vont alors s'attaquer aux membranes des cardiomyocytes, déclenchant des réactions de peroxydation lipidique. À l'appui de cette théorie, dite « $\varepsilon R O$ et fer », on trouve les résultats obtenus avec des animaux transgéniques surexprimant des gènes antioxydants tels que ceux codant la thiorédoxine 1 ou la métallothionéine, et dont le risque cardiaque est diminué par rapport aux animaux sauvages $[28,29]$. Cette toxicité de la DOX est indépendante de ses effets antitumoraux. Ceci laisse présager le développement d'agents cardioprotecteurs qui préviendraient les effets néfastes de la DOX sans affecter ses propriétés anticancéreuses [30].

\section{Conclusion}

Bien que le mot délétère soit encore trop souvent associé aux ERO, de nombreux travaux évoquent depuis quelques années leurs rôles bénéfiques. Les $\varepsilon R O$ ne sont pas seulement des radicaux destructeurs capables d'attaquer les constituants cellulaires, ce sont également des acteurs majeurs de la régulation métabolique et cellulaire de l'organisme. Le stress oxydant apparaît donc comme un mécanisme ubiquitaire dans lequel de nombreuses réactions physiologiques et pathologiques mettent en évidence une composante radicalaire. $\diamond$

\section{SUMMARY}

Reactive oxygen species and oxidative stress

Oxidative stress is defined as an imbalance between the production of reactive oxygen species (ROS) and the antioxidant capacity of the cell. For long, ROS have been considered as harmful by-products of the normal aerobic metabolism process of the mitochondria, implicated in a large variety of diseases. But there are now growing evidences that controlled ROS production also play physiological roles especially in regulating cell redox homeostasis and cell signaling. Biological ROS effects are now well documented. Data show that living organisms have not only adapted themselves to coexist with free radicals but have also developed mechanisms to use them advantageously. However their main sources and mechanisms of action remain poorly described. This review focuses on the main properties of ROS and their paradoxical effects. $\diamond$

\section{CONFLIT D'INTÉRÊTS}

Les auteurs déclarent n'avoir aucun conflit d'intérêts concernant les données publiées dans cet article.

\section{RÉFÉRENCES}

1. Commoner B, Townsend J, Pake GE. Free radicals in biological materials. Nature 1954 ; $174: 689-91$.

2. Harman D. Aging: a theory based on free radical and radiation chemistry. J Gerontol 1956; $11: 298-300$.

3. Guichard C, Pedruzzi $\varepsilon$, Fay M, et al. Les Nox/Duox : une nouvelle famille de NADPH oxydases. Med Sci (Paris) $2006 ; 22: 953-9$.

4. Sumimoto H. Structure, regulation and evolution of Nox-family NADPH oxidases that produce reactive oxygen species. FEBS / 2008 ; 275 : 3249-77.

5. Nauseef WM. Biological roles for the NOX family NADPH oxidases. J Biol Chem $2008 ; 283: 16961-5$

6. Brown DI, Griendling KK. Nox proteins in signal transduction. Free Radic Biol Med $2009 ; 47: 1239-53$.

7. Delattre J, Beaudeux JL, Bonnefont-Rousselot D. Radicaux libres et stress oxydant. Aspects biologiques et pathologiques. Cachan : Lavoisier, 2005.

8. Halliwell B, Gutteridge JMC. Free radicals in biology and medicine. $2^{e}$ ed. Oxford, UK : Clarendon, 1989.

9. Carrière $A$, Galinier $A$, Fernandez $Y$, et al. Les espèces actives de l'oxygène : le yin et le yang de la mitochondrie. Med Sci (Paris) 2006 ; $22: 47-53$.

10. Davies KJ. Protein damage and degradation by oxygen radicals. I. General aspects. J Biol Chem 1987 ; 262 : 9895-901.

11. Berlett BS, Stadtman ER. Protein oxidation in aging, disease, and oxidative stress. J Biol Chem 1997 ; 33 : 20313-6.

12. Finkel T. Redox-dependent signal transduction. FEBS Lett $2000 ; 476: 52-4$.

13. Levine RL. Carbonyl modified proteins in cellular regulation, aging, and disease. Free Radic Biol Med $2002 ; 32: 790-6$.

14. Murphy RC. Free radical-induced oxidation of glycerophosphocholine lipids and formation of biologically active products. Adv Exp Med Biol 1996; 416 : 51-8.

15. Dröge W. Free radicals in the physiological control of cell function. Physiol Rev $2002 ; 82: 47-95$.

16. Sundaresan $M, Y u$ ZX, Ferrans VJ, et al. Requirement for generation of $\mathrm{H}_{2} \mathrm{O}_{2}$ for platelet-derived growth factor signal transduction. Science $1995 ; 270$ : 296-9.

17. Marumo T, Schini-Kerth VB, Fisslthaler B, Busse R. Platelet-derived growth factor-stimulated superoxide anion production modulates activation of transcription factor NFK-B and expression of monocyte chemoattractant protein 1 in human aortic smooth muscle cells. Circulation $1997 ; 96: 2361-7$.

18. Jay DB, Papaharalambus CA, Seidel-Rogol B, et al. Nox5 mediates PDGFinduced proliferation in human aortic smooth muscle cells. Free Radic Biol Med $2008 ; 45: 329-35$

19. Bae YS, Kang SW, Seo MS, et al. Epidermal growth factor (EGF)-induced generation of hydrogen peroxide. J Biol Chem $1997 ; 272$ : 217-21.

20. Sundaresan M, Yu ZX, Ferrans VJ, et al. Regulation of reactive-oxygenspecies generation in fibroblasts by Racl. Biochem J 1996 ; $318: 379-82$. 
21. Stasia MJ. La granulomatose septique chronique $X^{+}$. Un fabuleux modèle d'étude de l'activation du complexe NADPH oxydase. Med Sci (Paris) $2007 ; 23: 526-32$

22. Varthaman $A$, Khallou-Laschet J, Thaunat 0 , et al. L'athérogenèse : une maladie dysimmunitaire. Med Sci (Paris) $2008 ; 24: 169-76$.

23. Morozova S, Suc-Royer I, Auwerx J. Modulateurs du métabolisme du cholestérol et avenir du traitement de l'athérosclérose. Med Sci (Paris) $2004 ; 20: 685-90$.

24. Poli G, Sottero B, Gargiulo S, Leonarduzzi G. Cholesterol oxidation products in the vascular remodeling due to atherosclerosis. Mol Aspects Med $2009 ; 30: 180-9$.

25. Von Hoff DD, Rozencweig M, Layard M, et al. Daunomycin-induced cardiotoxicity in children and adults. A review of 110 cases. Am J Med $1977 ; 62: 200-8$.

26. Simůnek T, Stérba M, Popelová 0 , et al. Anthracycline-induced cardiotoxicity: overview of studies examining the roles of oxidative stress and free cellular iron. Pharmacol Rep 2009 ; 61 : 154-71.

27. Myers C. The role of iron in doxorubicin-induced cardiomyopathy. Semin Oncol $1998 ; 25: 10-4$.

28. Shioji K, Kishimoto C, Nakamura $H$, et al. Overexpression of thioredoxin-1 in transgenic mice attenuates adriamycin-induced cardiotoxicity. Circulation $2002 ; 106: 1403-9$.
29. Sun X, Zhou Z, Kang YJ. Attenuation of doxorubicin chronic toxicity in metallothionein-overexpressing transgenic mouse heart. Cancer Res 2001 ; $61: 3382-7$.

30. Ludke AR, Al-Shudiefat AA, Dhingra S, et al. A concise description of cardioprotective strategies in doxorubicin-induced cardiotoxicity. Can J Physiol Pharmacol $2009 ; 87: 756-63$.

31. Mazat JP, Ransac $S$. Le complexe $b c_{1}$ de la chaîne respiratoire mitochondriale fonctionne selon l'hypothèse du cycle $\rho$ de Mitchell. La preuve par une approche stochastique? Med Sci (Paris) $2010 ; 26: 1079-86$.

TIRÉS À PART

C. Migdal

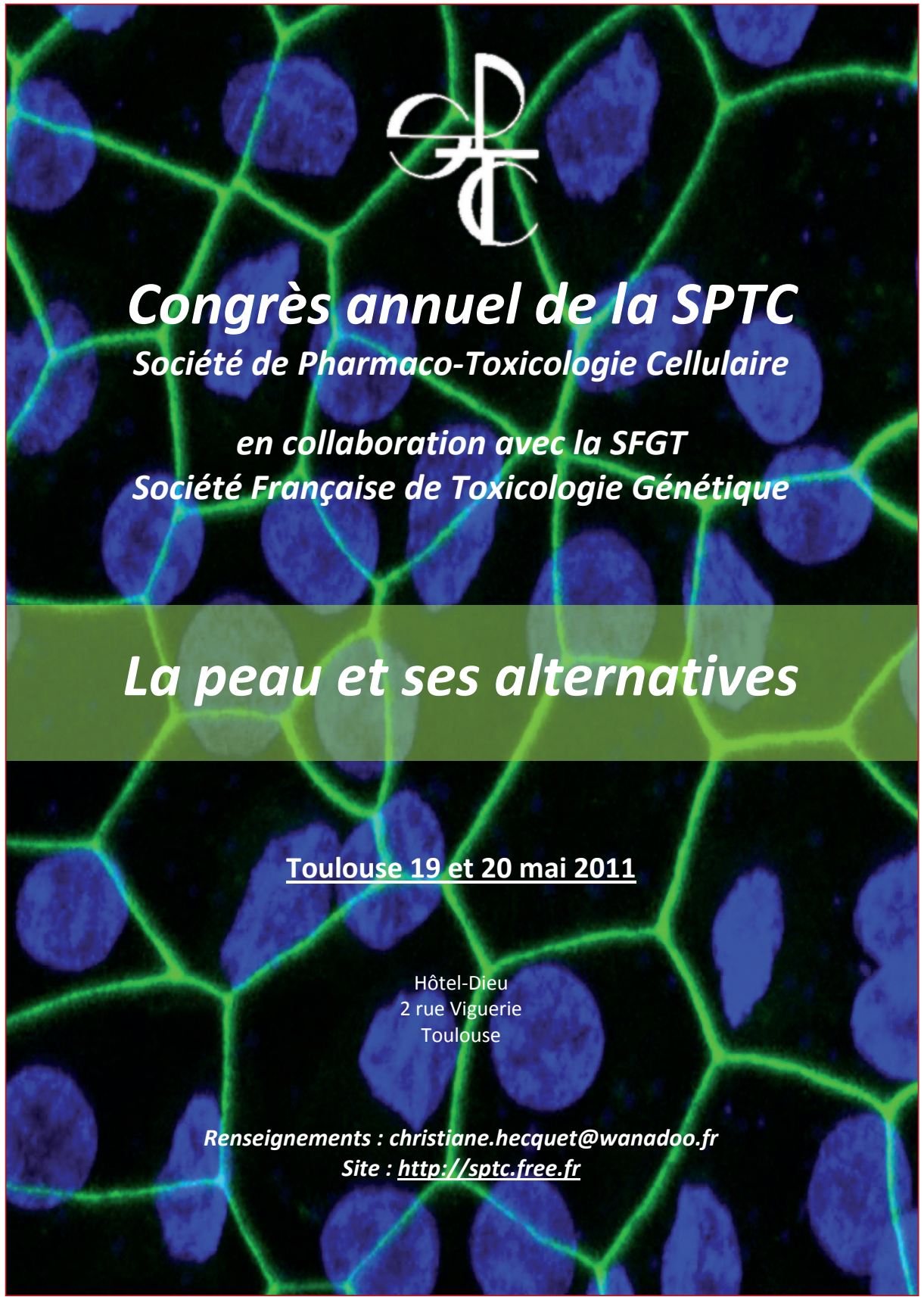

